\title{
Prehospital Factors Associated with Refractory Traumatic Arrest
}

\author{
Jeong Hun Lee $\mathbb{D}$, Yong Won Kim $\mathbb{D}^{D}$, Tae Youn Kim $\mathbb{D}$, Sanghun Lee $\mathbb{D}$, Han Ho Do $\mathbb{D}$, \\ Jun Seok Seo $\mathbb{D}$, and Seung Chul Lee $\mathbb{D}$
}

\author{
Department of Emergency Medicine, Dongguk University Ilsan Hospital, Dongguk University College of Medicine, Goyang, \\ Republic of Korea \\ Correspondence should be addressed to Yong Won Kim; chiefong@naver.com
}

Received 14 October 2021; Accepted 7 December 2021; Published 20 December 2021

Academic Editor: Roberto Cirocchi

Copyright $(2021$ Jeong Hun Lee et al. This is an open access article distributed under the Creative Commons Attribution License, which permits unrestricted use, distribution, and reproduction in any medium, provided the original work is properly cited.

Objective. Identification of the prehospital factors associated with a poor prognosis of immediate traumatic arrest should help reduce unwarranted treatment. We aim to reveal the clinical factors related to death after traumatic arrest on the scene. Methods. We performed a multicenter (4 tertiary hospitals in urban areas of South Korea) retrospective study on consecutive adult patients with trauma arrest on scene who were transferred by fire ambulance from January 2016 to December 2018. Patients with death on arrival in the emergency room (ER) were excluded. Prehospital data were collected from first aid records, and information on each patient's survival outcome in the ER was collected from an electronic database. Patients were divided into ER death and ER survival groups, and variables associated with prehospital trauma were compared. Results. A total of 145 (84.3\%) and 27 (15.7\%) patients were enrolled in the ER death and survival groups, respectively. Logistic regression analysis revealed that asystole (OR $4.033,95 \%$ CI $1.342-12.115, p=0.013)$ was related to ER death and that ROSC in the prehospital phase (OR 0.100, 95\% CI $0.012-0.839, p=0.034)$ was inversely related to ER death. In subgroup analysis of those who suffered fall injuries, greater height of fall was associated with ER death (15.0 (5.5-25.0) vs. 4.0 (2.0-7.5) meters, $p=0.001)$; the optimal height cutoff for prediction of ER death was 10 meters, with $66.1 \%$ sensitivity and $100 \%$ specificity. Conclusions. In cases of traumatic arrest, asystole, no prehospital ROSC, and falls from a greater height were associated with trauma death in the ER. Termination of resuscitation in traumatic arrest cases should be done on the basis of comprehensive clinical factors.

\section{Introduction}

Traumatic arrest immediately after injury is still usually irreversible and leads immediately to death despite the development of comprehensive modern trauma systems [1]. Immediate deaths account for about $60 \%$ of all traumarelated deaths. The overall survival rate from traumatic arrest on the prehospital phase is as low as 3.7\%, and previous studies reported very poor outcomes even following cardiopulmonary resuscitation (CPR) [2]. Therefore, establishment of termination of resuscitation (TOR) rules for cases of immediate traumatic arrest may avoid unnecessary consumption of valuable resources and unwarranted treatment. The 2012 National Association of Emergency Medical Service (EMS) Physicians and American College of Surgeons' Committee on Trauma (NAEMSP-ASCOT) published a joint position article concerning TOR rules for patients in traumatic cardiac arrest [3]. However, these TOR rules cannot be applied uniformly across different countries because they consider not only the characteristics of traumatic arrest but also the traumatic care system in the prehospital phase. We aim to reveal the clinical factors related to death after traumatic arrest on the scene and validate the objective protocol of early TOR rules for traumatic arrest patients according to a variety of situations.

\section{Methods}

2.1. Characteristics of the Local EMS. In the local EMS, in which this study was conducted, primary care to major trauma victims on-site or during transfer is provided by three paramedics who are members of the fire department. This local EMS had protocols for traumatic arrest that included performing CPR on scene for 4 minutes and then 
transporting the patient to the nearest emergency room with ongoing CPR in the ambulance until ROCS. Invasive care (such as advanced airway management, external defibrillation, and intravenous fluid administration) by paramedics is supervised online by emergency medical physicians (EMPs) as needed. Because of the medical laws in Korea, paramedics cannot declare death even if supervised online by EMPs, so trauma resuscitation must be provided to traumatic arrest patients during the prehospital phase if the patients do not have injuries that are incompatible with life or obvious signs of death.

2.2. Study Population and Design. This is a retrospective observational multicenter study. This study enrolled consecutive patients with traumatic arrest who were over 18 years of age, had no response with apnea, who were pulseless on scene, and who were transferred by EMS to one of four tertiary hospital ERs located in Gyeonggi province of the Republic of Korea from January 2016 to December 2018. Patients were excluded if they did not undergo any resuscitation efforts in the emergency room (ER), if they were pronounced dead on arrival (DOA) because of confirmed injuries incompatible with life or obvious signs of death (such as livor mortis or rigor mortis), or if they had a do not resuscitate (DNR) order. Prehospital data were collected from the first aid records of the paramedics; this included injury information and information on the invasive care that was provided (age, sex, injury mechanism, witness, bystander CPR, EMS CPR, return of spontaneous circulation (ROSC), initial arrest electrocardiogram (ECG) rhythm, time factors of transportation, advanced airway, external defibrillation, and intravenous fluid). ER survival outcome was collected from the NEDIS (National Emergency Department Information System) electronic database. Shockable rhythm was defined as ventricular fibrillation or ventricular tachycardia on the initial arrest ECG rhythm. ER death was defined as death in the ER despite extensive resuscitation efforts. ER survival was defined as survival after the ER visit and subsequent management (surgical operation, transfer to a trauma center, or admission to an intensive care unit). We compared prehospital traumaassociated variables between the ER death and ER survival groups.

2.3. Statistical Analysis. Continuous variables are presented as median values (interquartile range) and compared using the Mann-Whitney test. Nominal data were calculated as percentages based on the frequency of occurrence and compared using the chi-square or Fisher's exact test, as appropriate. Multivariate logistic regression was performed to identify prehospital trauma-associated variables related to ER death in order to suggest criteria for early TOR rules. The resulting odds ratios (ORs) are presented with 95\% confidence interval (95\% CI). A two-sided $p$ value of less than 0.05 is considered statistically significant. The statistical analyses were performed using IBM Statistical Package for the Social Sciences (SPSS) software version 24.0 (SPSS Inc., Chicago, IL, USA).

\section{Results}

During the study period, 221 adult traumatic arrest victims were transferred by EMS from the incident scene to one of four tertiary hospital ERs. Of these 221 patients, 29 were excluded from the study because they were declared DOA at the ER. Finally, 172 patients were enrolled in the study (Figure 1).

After acute trauma care in the ER, 145 (84.3\%) patients died in the ER, while the other 27 (15.7\%) survived and received subsequent management for trauma. In the ER death group, the time from hospital arrival to death was median 72 (IQR 56-97) minutes. The baseline characteristics of the study subjects are given in Table 1 .

Initial rhythm was different between the two groups. Asystole (59.3\% vs. 33.3\%) was more common in the ER death group than in the ER survival group, while PEA $(51.9 \%$ vs. $34.5 \%)$ and shockable rhythm (7.4\% vs. $3.4 \%)$ were more common in the ER survival group $(p=0.038)$. Regarding EMS management, defibrillation (14.8\% vs. 3.4\%) was more common in the ER survival group than in the ER death group. Prehospital ROSC (14.3\% vs. $1.4 \%)$ was more common in the ER survival group.

Among the 68 patients with injuries related to falls, 59 (86.8\%) belonged to the ER death group, and 9 (13.2\%) were in the ER survival group. Height of the fall was greater in the ER death group (median 15.0, IQR 5.5-25.0 m vs. median 4.0, IQR $2.0-7.5 \mathrm{~m}, p=0.001$ ).

There were no significant differences in age, sex, injury mechanism, witness, bystander CPR, EMS CPR, advanced airway, intravenous fluid, and time factors of the prehospital phase between the two groups.

The area under the receiver operating characteristic (ROC) curve describing the sensitivity and specificity of fall height for different cutoff levels was 0.838 (0.738-0.938) (Figure 2). The optimum height cutoff for prediction of trauma death at the emergency room was identified as $10 \mathrm{~m}$, with a sensitivity of $66.1 \%$ and a specificity of $100 \%$.

Multivariate analysis revealed that asystole (OR 4.033; $95 \%$ CI 1.342-12.115, $p=0.013$ ) was related to trauma death in the ER and that ROSC in the prehospital phase (OR 0.100; $95 \%$ CI $0.012-0.839, p=0.034)$ was inversely related to trauma death in the ER (Table 2).

When the early TOR rules consisted of asystole and no ROSC in the prehospital phase, the sensitivity was $58.6 \%$ (95\% CI 50.2-66.7\%), specificity was 70.37\% (49.8-86.3\%), positive predictive value was $91.4 \%$ (85.4-95.1\%), and negative predictive value was $24.1 \%$ (18.2-30.2\%) for trauma death in the ER.

\section{Discussion}

The TOR of traumatic arrest should be comprehensively determined by EMS providers or clinicians who take into consideration ethical issues, injury characteristics, condition of the patient, the local EMS system, and the overall medical environment. If there is no established guide to TOR, the point of termination of resuscitation may vary in accordance with the physician's opinion, even in similar situations. The 


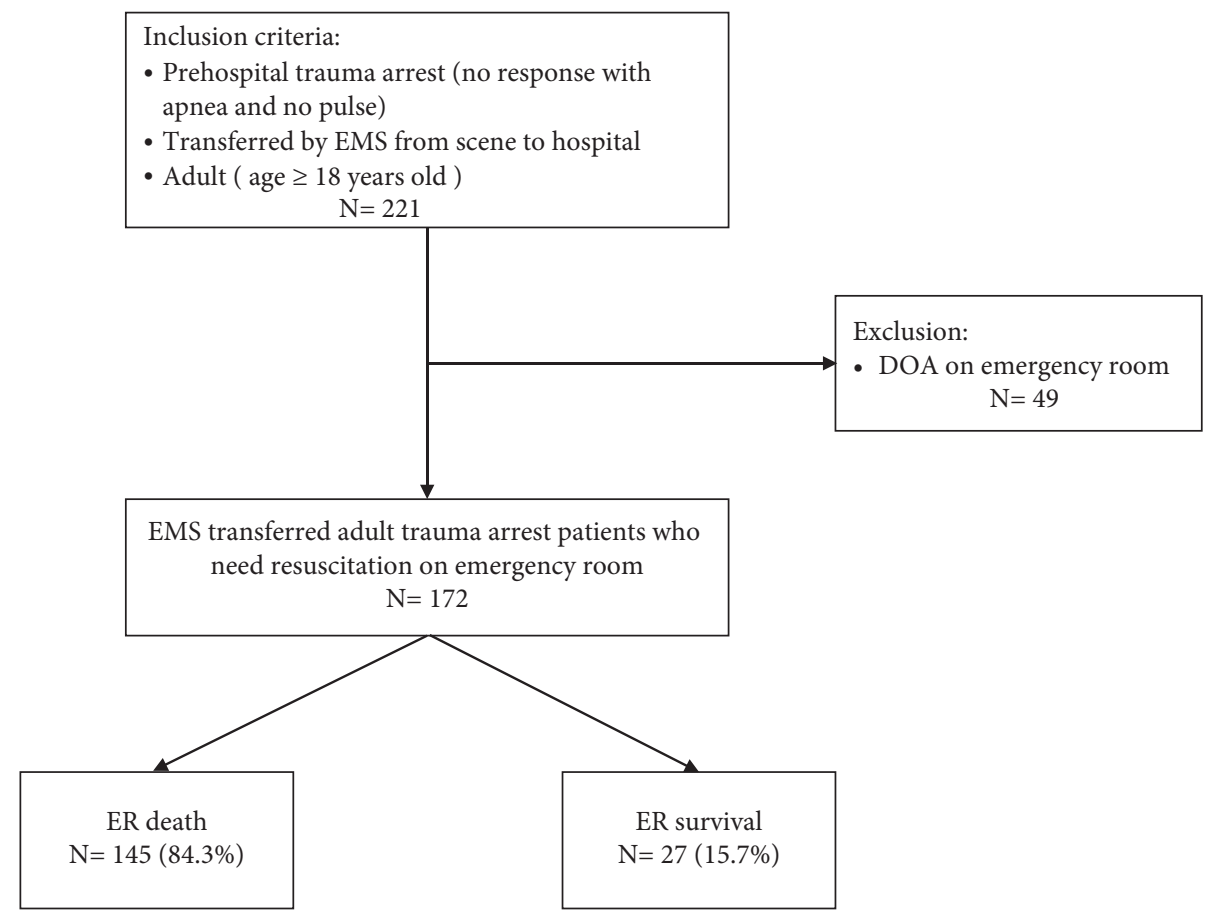

FIGURE 1: Flowchart of patient enrollment.

TABLE 1: Baseline characteristics in the ER death and ER survival groups.

\begin{tabular}{|c|c|c|c|c|}
\hline Parameters & Total, $N=172$ & ER death, $N=145(84.3 \%)$ & ER survival, $N=27(15.7 \%)$ & $P$ value \\
\hline Age (years) & $53(41-61) *$ & $54(39-63) *$ & $52(49-56) *$ & 0.099 \\
\hline Male, $N(\%)$ & $122(70.9)$ & $104(71.7)$ & $18(66.7)$ & 0.646 \\
\hline Injury mechanism & & & & 0.065 \\
\hline Blunt, $N(\%)$ & $169(98.3)$ & $144(99.3)$ & $25(92.6)$ & \\
\hline Penetration, $N(\%)$ & $3(1.7)$ & $1(0.7)$ & $2(7.4)$ & \\
\hline \multicolumn{5}{|l|}{ Detail injury mechanism } \\
\hline Fall down, $N(\%)$ & $68(39.5)$ & $59(40.7)$ & $9(33.3)$ & \\
\hline Pedestrian versus motor vehicle, $N(\%)$ & $41(23.8)$ & $35(24.1)$ & $6(22.2)$ & \\
\hline Motor vehicle occupant, $N(\%)$ & $29(16.9)$ & $22(15.2)$ & $7(25.9)$ & \\
\hline Motorcycle, $N(\%)$ & $17(9.9)$ & $17(11.7)$ & 0 & \\
\hline Other blunt injury, $N(\%)$ & $14(8.1)$ & $11(7.6)$ & $3(11.1)$ & \\
\hline Stab injury & $3(1.7)$ & $1(0.7)$ & $2(7.1)$ & \\
\hline Height of fall down (meter) & $\begin{array}{c}12.0(5.0-24.0) * \\
N=68\end{array}$ & $\begin{array}{c}15.0(5.5-25.0) * \\
N=59\end{array}$ & $\begin{array}{c}4.0(2.0-7.5) * \\
N=9\end{array}$ & 0.001 \\
\hline Witness & $84(48.8)$ & $70(48.3)$ & $14(51.9)$ & 0.835 \\
\hline Bystander CPR & $78(45.3)$ & $65(44.8)$ & $13(48.1)$ & 0.834 \\
\hline EMS CPR & $163(94.8)$ & $137(94.5)$ & $26(96.3)$ & 1.000 \\
\hline Initial rhythm & & & & 0.038 \\
\hline Asystole & $95(55.2)$ & $86(59.3)$ & $9(33.3)$ & \\
\hline PEA & $64(37.2)$ & $50(34.5)$ & $14(51.9)$ & \\
\hline VT or VF & $6(3.5)$ & $4(2.8)$ & $2(7.4)$ & \\
\hline Unknown & $7(4.1)$ & $5(3.4)$ & $2(7.4)$ & \\
\hline Advanced airway by EMS & $96(55.8)$ & $80(55.2)$ & $16(59.3)$ & 0.833 \\
\hline Shockable rhythm with defibrillation by EMS & $9(5.2)$ & $5(3.4)$ & $4(14.8)$ & 0.035 \\
\hline IV line with fluid resuscitation by EMS & $35(20.3)$ & $27(18.6)$ & $8(29.6)$ & 0.200 \\
\hline ROSC on prehospital phase & $6(3.5)$ & $2(1.4)$ & $4(14.3)$ & 0.006 \\
\hline Time from call to scene by EMS (min) & $8.0(6.0-11.0) *$ & $8.0(6.0-11.0) *$ & $8.0(6.0-10.5) *$ & 0.920 \\
\hline Time from scene to hospital by EMS (min) & $15.5(12.5-19.0) *$ & $15.0(12.5-19.0) *$ & $19.0(15.0-26.0) *$ & 0.222 \\
\hline Time from ER arrival to death (min) & & $72(56-97) *$ & & \\
\hline
\end{tabular}

${ }^{*}$ Median (interquartile range). ER, emergency room; CPR, cardiopulmonary resuscitation; EMS, emergency medical service; PEA, pulseless electrical activity; VT, ventricular tachycardia; VF, ventricular fibrillation; IV, intravenous; ROSC, return of spontaneous circulation. 


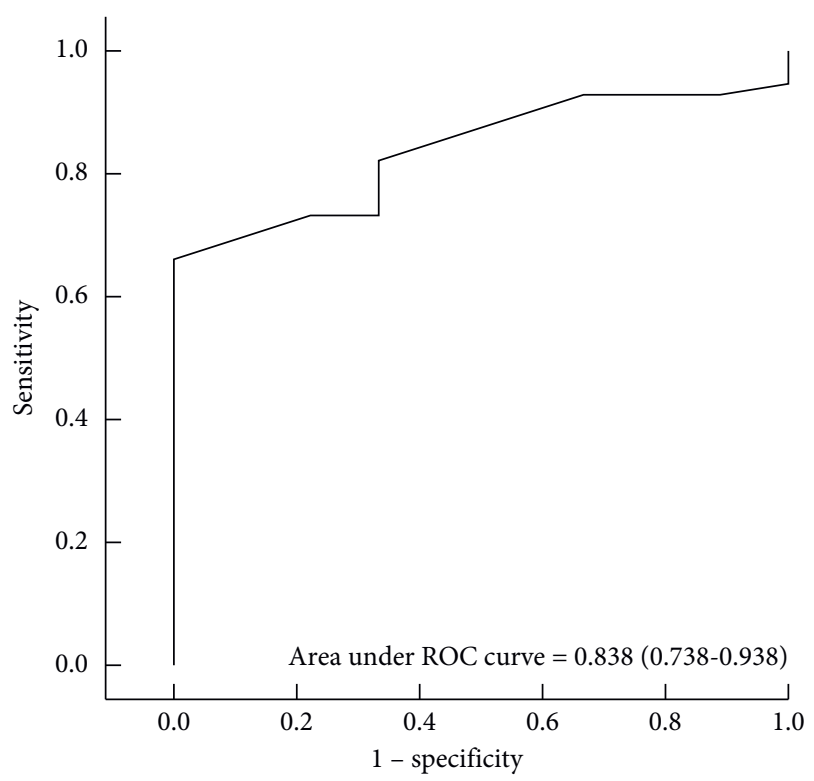

FIgURE 2: ROC curve for trauma death in the ER. The area under the curve for fall height was $0.838(0.738-0.938)$. The optimal fall height cutoff value for prediction of trauma death in the ER was 10 meters with a sensitivity of $66.1 \%$ and a specificity of $100 \%$. ROC, receiver operating characteristic; ER, emergency room.

TABLE 2: Multivariate analysis of prehospital factors related to ER death from traumatic arrest.

\begin{tabular}{lccc}
\hline Predictors of ER death & $\begin{array}{c}\text { Odds } \\
\text { ratio }\end{array}$ & $95 \%$ CI & $\begin{array}{c}P \\
\text { value }\end{array}$ \\
\hline Age (years) & 0.976 & $0.950-1.003$ & 0.085 \\
Male gender & 1.744 & $0.638-4.769$ & 0.278 \\
Witness & 1.623 & $0.556-4.735$ & 0.376 \\
Bystander CPR & 1.396 & $0.489-3.980$ & 0.533 \\
EMS CPR & 0.284 & $0.023-3.482$ & 0.325 \\
Blunt injury & 10.065 & $0.501-202.079$ & 0.131 \\
Asystole & 4.033 & $1.342-12.115$ & 0.013 \\
Supraglottic airway & 0.861 & $0.326-2.274$ & 0.763 \\
Need of defibrillation & 0.345 & $0.061-1.943$ & 0.228 \\
Fluid resuscitation & 0.487 & $0.159-1.488$ & 0.207 \\
ROSC on prehospital phase & 0.100 & $0.012-0.839$ & 0.034 \\
Duration of transfer by EMS & 0.974 & $0.920-1.031$ & 0.371 \\
(min) & & & \\
\hline
\end{tabular}

ER, emergency room; CI, confidence interval; CPR, cardiopulmonary resuscitation; EMS, emergency medical service; ROSC, return of spontaneous circulation.

region included in this study, Gyeonggi province of the Republic of Korea, has an area of approximately $10,000 \mathrm{~km}^{2}$ and a population of about 13 million and contains two trauma centers and six tertiary hospitals. Most patients with major trauma are transferred to the trauma center, but those with traumatic arrest are transferred to the nearest ER according to local EMS guidelines. All four tertiary hospitals in this study are located in urban areas, so the EMS arrival time at the scene and the time it took to get to the hospital were relatively short, approximately 8 minutes and 15 minutes, respectively. Since death cannot be declared during the prehospital phase under the current medical laws, the
EMS providers did not terminate resuscitation on-site or during transfer. Also, there were no gun shot injuries because it is illegal for ordinary people to carry guns in Korea, so there were fewer cases of penetrating injury than in other countries.

We found that asystole and no prehospital ROSC despite EMS CPR are the predictors of poor prognosis of traumatic arrest, and these factors may provide a suitable basis on which to determine early TOR on ER. Similarly, several previous studies have reported that asystole is a poor prognostic factor, and although the studies population of these studies (e.g., penetrating injury to thorax, military service-related trauma) differ from our study, the mortality rate of trauma arrest with asystole is to $87-100 \%$ [4-7]. According to NAEMSP-ACSCOT (3), asystole is identified as an important factor on which to determine whether to withhold resuscitation in both penetrating and blunt trauma, and no ROSC despite appropriate EMS treatment is proposed in the TOR protocol. Our findings further showed that traumatic arrest caused by a fall from a height of more than 10 meters may not respond to resuscitation efforts. In general, the greater the height from which a person falls, the more serious his or her injuries and the higher the mortality rate $[8,9]$. Thus, fall height is considered in most trauma centers as part of the criteria for trauma team activation [10]. Also, the reported mortality rates of falls from $\geq 6$ meters, $\geq 12$ meters, and $\geq 18$ meters are $22.7 \%, 50 \%$, and $100 \%$, respectively $[11,12]$. However, until now, little has been published about the prognosis in subgroups of patients who suffer from traumatic arrest at the scene due to a fall. Our findings showed that resuscitation efforts may be withheld or terminated if immediate traumatic arrest is caused by a fall from greater than 10 meters. Further studies may be needed that identify the situations in which resuscitation is futile in cases of traumatic arrest caused by other injury mechanisms.

Several studies have found that increases in EMS transfer time or in the duration of unsuccessful CPR are correlated with mortality in traumatic arrest, but it is difficult to suggest optimal cutoff time values for uniform TOR rules due to the heterogeneity of the study population [13-15]. Recently, the NAEMSP-ACSCOT suggested that up to 15 minutes of CPR should be provided before resuscitation efforts are terminated, but the evidence behind this suggestion remains unclear, and so they also suggested that further research is needed [3]. In our study, the median time from the scene to the hospital was 19.0 minutes (15.0-26.0) in the ER survival group; therefore, it may be inappropriate to terminate resuscitation efforts due to unsuccessful CPR even if the CPR time exceeds 15 minutes. Although not presented in our study, functional outcomes should also be considered when adding a time factor to the TOR rules because some past studies reported that longer CPR times were associated with poor neurological outcomes in cases of traumatic arrest even if the patient survived [16]. Factors that could prolong transport or CPR time include EMS management or hospital selection. Although the availability of EMS services generally improves the prognosis of major trauma, the survival outcomes following the provision of advanced life support by EMS providers remain controversial $[17,18]$. In our study, 
there was no significant difference between invasive trauma surveys and outcome when treatment was given by EMS personnel. There was some evidence that transfer to the trauma center results in a better prognosis in the case of major trauma and that a reduction in EMS transport time is a very important prognostic factor in cases of traumatic arrest [19]. Further studies are required to identify the risks and benefits of choosing between an acceptable transport time and an ideal hospital (trauma center vs. nearest nontrauma facility) in traumatic arrest patients.

The present study had some limitations. First, as this was a retrospective review study, because of a lack of information about the data sources, we could not quantify either injury severity using an injury severity score (ISS) or the amount of inhospital care that was provided, such as an emergency department thoracotomy. However, even if a prospective study is conducted, for patients who die in the emergency room due to traumatic arrest, an accurate ISS identification is difficult. Second, this study could not present long-term outcomes such as survival discharge and neurologic status. This is because the four institutions included in the study were not trauma centers, and the patients were often subjected to interfacility transfer for final treatment and rehabilitation.

\section{Conclusion}

In a trauma arrest patient on the scene, asystole, no prehospital ROSC, and fall from a greater height were associated with trauma death in the ER. The median transport time of ER survivors after traumatic arrest was 19 min, which was longer than the $15 \mathrm{~min}$, currently considered to be nonsalvageable. In urban areas of South Korea, if asystole and no ROSC in the prehospital phase are applied as early TOR rules, ER mortality could be predicted with a specificity of $70.37 \%$ and a positive predictive value of $91.4 \%$. In traumatic arrest, the TOR should be accompanied by decisions that take into account comprehensive clinical factors.

\section{Data Availability}

The data used to support the findings of this study are available from the corresponding author upon request.

\section{Ethical Approval}

This study was approved by the Institutional Review Board (IRB) of Dongguk University Ilsan Hospital, Dongguk University (approval number: DUIH 2020-03-060).

\section{Consent}

Informed consent was waived by the board.

\section{Conflicts of Interest}

The authors declare that they have no conflicts of interest.

\section{References}

[1] M. Gunst, V. Ghaemmaghami, A. Gruszecki, J. Urban, H. Frankel, and S. Shafi, "Changing Epidemiology of Trauma Deaths Leads to a Bimodal Distribution," Baylor University Medical Center Proceedings, vol. 23, no. 4, pp. 349-54, 2010.

[2] Z. T. Stockinger and N. E. McSwain Jr., "Additional evidence in support of withholding or terminating cardiopulmonary resuscitation for trauma patients in the field 1 1This article was written by LCDR Zsolt T Stockinger, MC, USNR, while a fellow at Tulane University Health Sciences Center training in trauma surgery and surgical critical care. The views expressed in this article are those of the author and do not reflect the official policy or position of the Department of the Navy, Department of Defense, or the United States government," Journal of the American College of Surgeons, vol. 198, no. 2, pp. 227-231, 2004.

[3] M. G. Millin, S. M. Galvagno, S. R. Khandker, A. Malki, E. M. Bulger, and A. C. Surgery, "Withholding and termination of resuscitation of adult cardiopulmonary arrest secondary to trauma," Journal of Trauma and Acute Care Surgery, vol. 75, no. 3, pp. 459-467, 2013.

[4] S. J. Stratton, K. Brickett, and T. Crammer, "Prehospital pulseless, unconscious penetrating trauma victims," The Journal of Trauma, Injury, Infection, and Critical Care, vol. 45, no. 1, pp. 96-100, 1998.

[5] C. Aprahamian, J. C. Darin, B. M. Thompson, J. R. Mateer, and J. F. Tucker, "Traumatic cardiac arrest: scope of paramedic services," Annals of Emergency Medicine, vol. 14, no. 6, pp. 583-586, 1985.

[6] N. T. Tarmey, C. L. Park, O. J. Bartels, T. C. Konig, P. F. Mahoney, and A. J. Mellor, "Outcomes following military traumatic cardiorespiratory arrest: a prospective observational study," Resuscitation, vol. 82, no. 9, pp. 1194-1197, 2011.

[7] M. J. Seamon, C. A. Fisher, J. P. Gaughan, H. Kulp, D. T. Dempsey, and A. J. Goldberg, "Emergency department thoracotomy: survival of the least expected," World Journal of Surgery, vol. 32, no. 4, pp. 604-612, 2008.

[8] K. Turgut, M. E. Sarihan, C. Colak, T. Güven, A. Gür, and S. Gürbüz, "Falls from height: a retrospective analysis," World Journal of Emergency Medicine, vol. 9, no. 1, p. 46, 2018.

[9] F. Lapostolle, C. Gere, S. W. Borron et al., "Prognostic factors in victims of falls from height," Critical Care Medicine, vol. 33, no. 6, pp. 1239-1242, 2005.

[10] T. Kristiansen, K. Søreide, K. G. Ringdal et al., "Trauma systems and early management of severe injuries in Scandinavia: review of the current state," Injury, vol. 41, no. 5, pp. 444-452, 2010.

[11] R. F. Buckman Jr. and P. D. Buckman, "Vertical deceleration trauma: principles of management," Surgical Clinics of North America, vol. 71, no. 2, pp. 331-344, 1991.

[12] C.-C. Liu, C.-Y. Wang, H.-C. Shih et al., "Prognostic factors for mortality following falls from height," Injury, vol. 40, no. 6, pp. 595-597, 2009.

[13] R. L. Fulton, W. J. Voigt, and A. S. Hilakos, "Confusion surrounding the treatment of traumatic cardiac arrest," Journal of the American College of Surgeons, vol. 181, no. 3, pp. 209-214, 1995.

[14] D. Powell, E. E. Moore, C. C. Cothren et al., "Is emergency department resuscitative thoracotomy futile care for the critically injured patient requiring prehospital cardiopulmonary resuscitation?" Journal of the American College of Surgeons, vol. 199, no. 2, pp. 211-215, 2004. 
[15] Y. Moriwaki, M. Sugiyama, T. Yamamoto et al., "Outcomes from prehospital cardiac arrest in blunt trauma patients," World Journal of Surgery, vol. 35, no. 1, pp. 34-42, 2011.

[16] T. J. Esposito, G. J. Jurkovich, C. L. Rice, R. V. Maier, M. K. Copass, and D. G. Ashbaugh, "Reappraisal of emergency room thoracotomy in a changing environment," The Journal of Trauma, Injury, Infection, and Critical Care, vol. 31, no. 7, pp. 881-887, 1991.

[17] J. P. Ornato, E. J. Craren, N. M. Nelson, and K. F. Kimball, "Impact of improved emergency medical services and emergency trauma care on the reduction in mortality from trauma," The Journal of Trauma, Injury, Infection, and Critical Care, vol. 25, no. 7, pp. 575-579, 1985.

[18] M. Eckstein, L. Chan, A. Schneir, R. Palmer, and A. C. Surgery, "Effect of prehospital advanced life support on outcomes of major trauma patients," The Journal of Trauma, Injury, Infection, and Critical Care, vol. 48, no. 4, pp. 643-648, 2000.

[19] T. Garwe, L. D. Cowan, B. Neas, T. Cathey, B. C. Danford, and P. Greenawalt, "Survival benefit of transfer to tertiary trauma centers for major trauma patients initially presenting to nontertiary trauma centers," Academic Emergency Medicine, vol. 17, no. 11, pp. 1223-1232, 2010. 